PESQUISA

\title{
ADEQUAÇÃO NUTRICIONAL DOS CARDÁPIOS DOS CENTROS MUNICIPAIS DE EDUCAÇÃO INFANTIL DE GOIÂNIA-GO
}

\section{NUTRITIONAL ADEQUACY OF THE MENUS OF THE MUNICIPAL CENTERS FOR EARLY CHILDHOOD EDUCATION IN GOIÂNIA-GO}

\author{
Eurismar Cardoso da Silva1 \\ Pontifícia Universidade Católica de Goiás. Escola de Ciências Sociais e da Saúde. Curso de \\ Nutrição. Goiânia, Goiás, Brasil. \\ https://orcid.org/0000-0002-2117-4638 \\ eurisnutri1974@outlook.com
}

Profa. Orientadora Me. Nair Augusta de Araújo Almeida Gomes ${ }^{2}$

Pontifícia Universidade Católica de Goiás. Escola de Ciências Sociais e da Saúde. Curso de Nutrição. Goiânia, Goiás, Brasil. https://orcid.org/0000-0001-6882-0388 nairaugustaalmeida@yahoo.com.br

\begin{abstract}
1 Participação na formulação e elaboração do artigo. Desenho do Projeto, Escrita - Primeira Redação, Metodologia, Discussão dos Resultados, Escrita - Revisão versão final.

${ }^{2}$ Participação na formulação e elaboração do artigo. Desenho do Projeto, Escrita - Primeira Redação, Metodologia, Discussão dos Resultados, Escrita - Revisão e aprovação da versão final.
\end{abstract}

RESUMO: O presente estudo objetivou avaliar a adequação da composição nutricional dos cardápios dos Centros Municipais de Educação Infantil (CMEI) de Goiânia aos parâmetros propostos pelo Programa Nacional de Alimentação Escolar (PNAE). A pesquisa consistiu-se na análise da composição nutricional dos cardápios, ofertados por um período de onze meses (janeiro a dezembro de 2019), às crianças da faixa etária de 12 meses a três anos de idade, dos $138 \mathrm{CMEl}$ de Goiânia-GO. Para fins de comparação dos valores encontrados foram utilizadas as referências de energia, macronutrientes e micronutrientes do programa, para a faixa etária selecionada. Com base nas recomendações do PNAE, os cardápios foram em média insuficientes em relação à fibra alimentar e com valor muito próximo ao recomendado para o ferro. Por outro lado, para o Magnésio, Vitamina $A$ e Vitamina $\mathrm{C}$ as quantidades consumidas, em média, foram de duas a seis vezes maiores que as recomendadas. A partir dos resultados encontrados evidenciou-se a necessidade da oferta de mais frutas e vegetais nos cardápios diários, para que o teor de fibras seja alcançado e favorecer o desenvolvimento nas crianças do hábito de inclui-los em sua alimentação diária, da infância à vida adulta. E assim, garantir o Direito Humano à Alimentação Adequada e, consequentemente, a promoção da saúde. Destaca-se a importância do profissional nutricionista, na elaboração do cardápio, a fim de garantir a adequação da alimentação oferecida em termos qualitativos e quantitativos, evitando carências ou excessos nutricionais, 
contribuindo, para o crescimento, desenvolvimento e formação de hábitos alimentares saudáveis das crianças.

Palavras-chave: Alimentação Escolar. Planejamento de cardápio. Recomendações nutricionais.

ABSTRACT: This study aimed to evaluate the adequacy of the nutritional composition of the menus of the Municipal Centers for Early Childhood Education (CMEI) of Goiânia to the parameters proposed by the National School Feeding Program (PNAE). The research consisted in the analysis of the nutritional composition of the menus, offered for a period of eleven months (January to December 2019), to children aged 12 months to three years old, of the $138 \mathrm{CMEI}$ of Goiânia-GO. To the purpose of comparing the values found, the references of energy, macronutrients, and micronutrients of the PNAE were used, for the selected age group. Based on the PNAE recommendations, the menu was, on average, insufficient in relation to dietary fiber and with a value close to that recommended for iron. On the other hand, for Magnesium, Vitamin A and Vitamin C the amounts consumed, on average, were about two to six times greater than those recommended. From the results found, it is evident the need to offer more fruits and vegetables in the daily menus, so that the fiber content is reached and to favor the development of children in the habit of including them in their daily diet, from childhood life adult. And so, to guarantee the Human Right to Adequate Food and, consequently, health promotion. The importance of the professional nutritionist in the elaboration of the menus is highlighted, to guarantee the adequacy of the food offered in qualitative and quantitative terms, avoiding nutritional deficiencies or excesses, thus contributing to the growth, development and formation of health eating habits of the children.

Keywords: School Feeding. Menu Planning. Recommended Dietary Allowances.

\section{INTRODUÇÃO}

Visando a promoção de uma alimentação adequada e saudável como garantia da Segurança Alimentar e Nutricional (SAN), o Programa Nacional de Alimentação Escolar (PNAE), propõe a oferta de refeições às crianças matriculadas nas instituições públicas e filantrópicas do País (BRASIL, 2013).

O PNAE, instituído em 1955, possui como objetivo primário contribuir para 0 crescimento e 0 desenvolvimento biopsicossocial, a aprendizagem, o rendimento escolar e a formação de hábitos alimentares saudáveis, por meio de ações de educação alimentar e nutricional e da oferta de refeições que cubram as necessidades nutricionais diárias preconizadas (BRASIL, 2009). Para tanto, considera-se a necessidade de aproximação dos cardápios da alimentação escolar aos hábitos alimentares dessas crianças enquanto estratégia para favorecer o consumo das preparações servidas (PAIVA; FREITAS; SANTOS, 2016).

A normativa que dispõe sobre 0 atendimento da alimentação escolar, estabelece que os cardápios fornecidos às crianças que frequentam os Centros de Educação Infantil (CEI) em período integral, elaborados por nutricionistas, devem contemplar no mínimo $70 \%$ dos requerimentos nutricionais diários, 
distribuídas em pelo menos três refeições (BRASIL, 2013). Adicionalmente, essa alimentação desempenha papel de relevância social, uma vez que, em muitos casos, é considerada como a principal refeição e fonte de nutrição do dia (RABELO et al.,2012).

Dentre as diretrizes do programa destaca-se o emprego de uma alimentação saudável e adequada, que compreende o uso de alimentos variados, seguros, que respeite as tradições locais e os hábitos alimentares saudáveis, em conformidade com a faixa etária e o estado de saúde dos educandos (BRASIL, 2009).

Os CEI possuem papel fundamental na saúde e alimentação das crianças, pois, muitas começam a frequentar tais instituições desde os primeiros anos de vida e lá permanecem por longos períodos do dia. A interação com educadores, manipuladores de alimentos e outras crianças irá influenciar nas escolhas e práticas alimentares delas (SBP, 2008).

Em face desse cenário, é importante avaliar a adequação nutricional dos cardápios dos Centros Municipais de Educação Infantil (CMEI) de Goiânia, no intuito de verificar 0 atendimento dos parâmetros propostos pelo PNAE e disponibilizar tais informações aos técnicos e profissionais do programa, seja no âmbito da gestão e/ou da escola.

\section{METODOLOGIA}

Trata-se de um estudo do tipo transversal e descritivo, com característica quantitativa, desenvolvido no município de Goiânia-GO, durante o ano de 2020.

Os dados utilizados nessa pesquisa, os cardápios planejados e com a composição nutricional previamente calculada pelo quadro técnico de nutricionistas da Secretaria Municipal de Educação (SME) (com as preparações/dia da semana), foram cedidos (dados secundários) pela SME, sendo assinado o termo de Fiel Depositário e dispensada a submissão ao Comitê de Ética em Pesquisa. Para a análise da composição nutricional dos cardápios, as nutricionistas consideraram o per capita dos alimentos que compõem as preparações constantes nos cardápios. Foi utilizado o software @Excel, tendo como base de dados para a análise nutricional a Tabela de Composição Nutricional e o Instituto Brasileiro de Geografia e Estatística (TACO, 2011; IBGE, 2011).

As crianças atendidas nos CMEl em tempo integral realizam cinco refeições diárias (desjejum, colação, almoço, lanche da tarde e jantar) de modo a suprir no mínimo $70 \%$ das necessidades nutricionais. $O$ estudo consistiu na análise da adequação da composição nutricional dos cardápios, ofertados por um período de onze meses (janeiro a dezembro de 2019), às crianças da faixa etária de 12 meses a três anos de idade, dos 138 Centros Municipais de Educação Infantil (CMEI) de Goiânia-GO. Para tal, obteve-se 
a média de valores de energia, macronutrientes, micronutrientes e fibra dos cardápios dos meses de janeiro a dezembro. Para fins de comparação dos valores encontrados foram utilizadas as referências do PNAE, quadro I (BRASIL, 2013).

Quadro 1 - Valores de referência de energia, macro e micronutrientes utilizados pelo Programa Nacional de Alimentação Escolar, para crianças da faixa etária de 1 a 3 anos.

\begin{tabular}{|c|c|c|c|c|c|c|c|c|c|c|}
\hline \multicolumn{7}{|c|}{$70 \%$ das Necessidades Nutricionais diárias } \\
\hline Energia & HCO & Ptn & Lipídios & Fibras & \multicolumn{2}{|c|}{ Vitaminas } & \multicolumn{3}{|c|}{ Minerais $(\mathrm{mg})$} \\
\cline { 6 - 11 }$($ Kcal $)$ & $(\mathrm{g})$ & $(\mathrm{g})$ & $(\mathrm{g})$ & $(\mathrm{g})$ & $\mathrm{A}(\mu \mathrm{g})$ & $\mathrm{C}(\mathrm{mg})$ & $\mathrm{Ca}$ & $\mathrm{Fe}$ & $\mathrm{Mg}$ & $\mathrm{Zn}$ \\
& & & & & & & & & & \\
\hline 700 & 114,9 & 21,9 & 17,5 & 13,3 & 210 & 12 & 350 & 4,9 & 56 & 2,1 \\
\hline
\end{tabular}

Fonte: Programa Nacional de Alimentação Escolar- PNAE.

Realizaram-se análises descritivas dos dados obtidos, utilizando-se planilhas Excel Microsoft 2010 (For Windows 7), gerando um banco de dados do qual geraram-se os quadros e tabelas que integram o estudo.

\section{RESULTADOS E DISCUSSÃO}

Segundo o Fundo Nacional de Desenvolvimento da Educação (FNDE), seis micronutrientes devem ser priorizados nas refeições escolares, sendo eles: cálcio, ferro, magnésio, zinco, vitaminas $A$ e $C$ (BRASIL, 2013). A análise da composição de energia, macronutrientes, micronutrientes e fibra dos cardápios, bem como, a adequação aos requerimentos nutricionais recomendados pelo FNDE, estão descritos na tabela 1.

Tabela 1 - Média de valores de energia e nutrientes dos cardápios dos meses de janeiro a dezembro e percentual de adequação às referências utilizadas pelo Programa Nacional de Alimentação Escolar em CMEI de Goiânia, Goiás, 2019.

Cardápios CMEI

\begin{tabular}{cccccccccccc}
\hline $\begin{array}{c}\text { Meses/ } \\
2019\end{array}$ & $\begin{array}{c}\text { Calorias } \\
(\mathrm{kcal})\end{array}$ & $\begin{array}{c}\text { PTN } \\
(\mathrm{g})\end{array}$ & $\begin{array}{c}\text { CHO } \\
(\mathrm{g})\end{array}$ & $\begin{array}{c}\text { LIP } \\
(\mathrm{g})\end{array}$ & $\begin{array}{c}\text { Fibras } \\
(\mathrm{g})\end{array}$ & $\begin{array}{c}\mathrm{Ca} \\
(\mathrm{mg})\end{array}$ & $\begin{array}{c}\text { Fe } \\
(\mathrm{mg})\end{array}$ & $\begin{array}{c}\mathrm{Zn} \\
(\mathrm{mg})\end{array}$ & $\begin{array}{c}\mathrm{Mg} \\
(\mathrm{mg})\end{array}$ & $\begin{array}{c}\text { Vit. A } \\
(\mu \mathrm{g})\end{array}$ & $\begin{array}{c}\text { Vit. C } \\
(\mathrm{mg})\end{array}$ \\
\hline 1 e 2 & 797,60 & 31,10 & 120,20 & 21,40 & 9,60 & 321,00 & 4,30 & 3,70 & 125,20 & 620,90 & 69,80 \\
3 & 805,90 & 31,00 & 119,00 & 22,90 & 9,80 & 325,10 & 4,40 & 3,70 & 131,30 & 626,40 & 73,50 \\
4 & 771,2 & 31,15 & 117,7 & 19,55 & 9,85 & 373,3 & 4,05 & 3,2 & 112,6 & 570,7 & 63,8 \\
5 & 808,75 & 31,6 & 123,35 & 21 & 10,75 & 383,65 & 4,45 & 3,35 & 119,25 & 544,85 & 69,15
\end{tabular}




\begin{tabular}{cccccccccccc}
\hline 6 & 884 & 35 & 127,7 & 23,4 & 10,2 & 388,05 & 4,5 & 3,6 & 120,25 & 531,95 & 63,75 \\
8 & 803,1 & 31,35 & 122,75 & 20,75 & 10,6 & 379,8 & 4,3 & 3,25 & 122,1 & 576,75 & 73,45 \\
9 & 823,6 & 32 & 126,5 & 21,1 & 11,1 & 373,65 & 4,4 & 3,95 & 141,1 & 660,25 & 84,5 \\
10 & 799,1 & 31,2 & 118,35 & 22,3 & 9,95 & 349 & 4,05 & 3,6 & 128,85 & 671,15 & 104,55 \\
11 & 833,45 & 32,5 & 124 & 23,05 & 10,25 & 393,35 & 4,4 & 4 & 136 & 667,65 & 86,65 \\
12 & 794,25 & 31,35 & 120,25 & 20,85 & 10,55 & 328,85 & 4,4 & 3,85 & 134,4 & 671,95 & 87,9 \\
MÉDIA & 812,10 & 31,83 & 121,98 & 21,63 & 10,27 & 361,58 & 4,33 & 3,62 & 127,11 & 614,26 & 77,71 \\
PNAE & 700 & 21,9 & 114,9 & 17,5 & 13,3 & 350 & 4,9 & 2,1 & 56 & 210 & 12 \\
Adeq.\% & 116,01 & 145,34 & 106,16 & 123,6 & 77,22 & 103,31 & 88,37 & 172,38 & 226,98 & 292,5 & 647,58 \\
\hline
\end{tabular}

Fonte: Elaborado pelas autoras, 2020.

Com base nas recomendações do PNAE, as preparações constantes nos cardápios e consumidas pelas crianças de 1 a 3 anos, foram em média, menores que o estabelecido em relação à fibra alimentar e com valores próximos ao recomendado para o ferro. Por outro lado, para o Magnésio, Vitamina A e Vitamina $C$ as quantidades foram cerca de duas a seis vezes maiores que as recomendadas.

O consumo adequado de energia, macronutrientes e micronutrientes é requerido pela participação deles nos mecanismos biológicos, crescimento e desenvolvimento cognitivo e estado imunológico dos escolares (ISSA et al., 2014). Por outro lado, a oferta insuficiente de energia e nutrientes comprometem 0 estado nutricional, a aprendizagem, o desenvolvimento e o desempenho escolar (LEÃO et al., 2018).

No estudo realizado por Souza e colaboradores (2018) em CEI de Maceió/AL, a energia ofertada correspondeu a $18,3 \%$ do total previsto pelo PNAE, para o atendimento em período integral. Para os autores o alcance da meta de $100 \%$ das necessidades energéticas durante o dia alimentar da criança é pouco praticável. Pois, embora seja previsto o compartilhamento da responsabilidade alimentar com os pais, para completar as necessidades nutricionais diárias, pode-se pressupor a impossibilidade da garantia do suprimento energético que deixou de ser consumido na instituição educacional (SOUZA et al., 2018). Diferentemente do resultado desse estudo que alcançou $100 \%$ de adequação às recomendações do PNAE nesse quesito.

A oferta de cardápios balanceados é importante na promoção de hábitos alimentares saudáveis e adequados, favorece o maior consumo de frutas, verduras e legumes, reduz a ingestão de produtos processados e ultraprocessados, auxilia no controle de deficiências nutricionais, além de fomentar a economia local através da aquisição de produtos da 
agricultura familiar (BRASIL, 2009; BRASIL, 2013; BRASIL, 2014).

Embora o PNAE forneça diretrizes regulatórias para a elaboração dos cardápios, os nutricionistas devem levar em conta vários aspectos que não são definidos pela legislação. A exemplo da disponibilidade de alimentos, técnicas de preparo e combinações de ingredientes, cores e formas e gerenciamento e execução (cronograma de entrega de fornecedores, infraestrutura escolar, tempo e dificuldade no preparo e custo e recursos financeiros) (LEÃO et al., 2018; BIANCHINI et al., 2020).

O número insuficiente de nutricionistas no quadro técnico e de manipuladores de alimentos para a demanda, a infraestrutura da instituição educacional (espaço físico), equipamentos, utensílios e recursos financeiros, estão entre as principais barreiras para que não se atinja a qualidade nutricional da refeição (PROENÇA et al., 2008; BIANCHINI et al., 2020).

Os baixos valores de fibras encontrados no presente estudo se assemelham aos relatados por Inque et al. (2015), em CMEI do município de Colombo, Paraná, no qual a mediana ficou abaixo do valor de referência. Bem como, por Almeida e colaboradores (2015) na avaliação do consumo alimentar em unidades de educação infantil do município de Belo Horizonte (MG) e por Souza et al. (2018) em CEl de Maceió (AL), com a adequação percentual do consumo de fibra alimentar variando de 9,7 a $30,9 \%$.

As fibras alimentares são compostos vegetais, essenciais para 0 correto funcionamento dos intestinos. Algumas diminuem a absorção de colesterol, gorduras e açúcares, pois permanecem no estômago por mais tempo, fazendo com que se prolongue a sensação de saciedade (PACHECO; CHAVES, 2013).

A fibra desempenha um papel fundamental na saúde do organismo, em quantidades adequadas, é responsável pelo controle de peso, prevenção de problemas circulatórios e hipertensão, câncer de cólon e diabetes (BERNAUD; RODRIGUES, 2013).

A oferta de alimentos fontes de fibra alimentar, em unidades escolares de atendimento em período integral, será obrigatoriamente, no mínimo $520 \mathrm{~g} /$ estudantes/semana de frutas in natura, legumes e verduras, assim distribuídos, frutas in natura, no mínimo, quatro dias por semana e hortaliças, no mínimo, cinco dias por semana (BRASIL, 2020a).

No presente estudo verificou-se que os valores do ferro embora próximos, não atingiram a recomendação para a faixa etária considerada. Lopes et al. (2015) analisaram o teor de ferro de alimentos ofertados nos cardápios de creches do extremo sul de Santa Catarina. Apenas para as crianças de 1 a 3 anos se atingiu a recomendação de $70 \%$ da necessidade 
diária de ferro. A adequação ao ferro também foi inferior ao preconizado no estudo de Frampton et al. (2014). Os valores abaixo da recomendação contribuem para o alto índice de anemia ferropriva.

Para Souza e colaboradores (2018), a inadequação de ferro pode ser explicada pela baixa frequência de consumo e reduzida porção per capita de carne, alimento considerado a melhor fonte deste nutriente. Tal constatação é preocupante visto que a anemia ferropriva é uma carência que acomete um percentual significante de crianças menores de 5 anos em âmbito mundial (BLACK et al., 2013).

O consumo de vitamina $C$ encontrado neste estudo corrobora os valores apresentados na pesquisa realizada por Souza e colaboradores (2018), na qual a oferta e consumo da vitamina $C$ foi cerca de 5 a 6 vezes superior ao estipulado e cerca de duas vezes no estudo de Leão e colaboradores (2018) para as crianças do berçário.

Embora o consumo acima da recomendação não represente risco toxicológico, a ingestão em quantidade suficiente de vitamina C favorece a absorção de ferro não heme da dieta e o adequado funcionamento do sistema imunológico da criança (ABRANCHES et al., 2009). Já o consumo de vitamina $C$ abaixo da recomendação pode prejudicar o processo de cicatrização, formação dos dentes e ossos e a integridade dos capilares (BERTIN et al., 2008; CONCEIÇÃO et al., 2010).

Os valores encontrados para a vitamina A nessa investigação foram mais que duas vezes acima das recomendações. Diferentemente dos percentuais de adequação observados para crianças menores de cinco anos, nos estudos realizados em CEI no estado de Alagoas por Souza et al. (2018) e por Ferreira et al. (2013). Fato preocupante, em se considerando que a deficiência de vitamina A se destaca como uma das carências nutricionais mais prevalentes no país (BLACK et al., 2013). O consumo elevado de vitamina $A$ pode estar relacionado a ingestão de alimentos fontes desse nutriente, como alguns legumes (cenoura, abóbora) e frutas (mamão), além do consumo de leite de vaca (INQUE et al., 2015).

No que concerne ao zinco, constatou-se entre as crianças de 1 a 3 anos oferta superior a recomendação. Para o Zinco, os valores observados ultrapassaram as recomendações do PNAE nos estudos realizados por Souza et al. (2018), Almeida et al. (2015) e por Leão et al. (2018), no qual a porcentagem de zinco alcançada foi de $304,8 \%$. Estimativas indicam que 17\% da população mundial estão em risco de deficiência de zinco, considerada um problema de saúde pública frequentemente subnotificado, e que causa deficiência no sistema imunológico, se associa ao retardo do crescimento, 
cognitivo e distúrbio neurossensorial (BLACK et al., 2013).

Para o magnésio, as quantidades foram maiores que as recomendadas. Corroborando os resultados encontrados no estudo de Almeida et al. (2015). O magnésio, que está presente nos ossos, tecidos moles, músculos e fluídos corpóreos é de extrema importância e atua como cofator em mais de 300 sistemas enzimáticos (LEAL et al., 2015).

Após sessenta e cinco anos de existência do PNAE, ainda prevalece o desafio em garantir que os nutrientes sejam ofertados em quantidades adequadas, por meio de alimentos que atendam aos princípios de uma alimentação segura e saudável (BRASIL, 2020b). Com vistas a alcançar a alimentação escolar que atenda aos requerimentos nutricionais do programa, requer-se a adequada elaboração dos cardápios.

O acompanhamento da execução do PNAE, pode ser realizado diretamente pela sociedade, por meio do Conselho de Alimentação Escolar (CAE), FNDE, Conselhos de Segurança Alimentar e Nutricional Estadual e Municipal (Conesan e Consea) e pelo Ministério Público: Instâncias que podem detectar inconsistências e pautar o debate, em busca de soluções. Isso implica em dar sugestões e propor ajustes aos cardápios, subsidiar a formulação de propostas de inovações no cardápio escolar atualmente praticado, adequando-o às prerrogativas do PNAE e expectativas dos educandos (BRASIL, 2015).

O estado nutricional na infância está relacionado com a predisposição para a aprendizagem e é importante no funcionamento cerebral e na capacidade da memória (MOREIRA et al., 2015).

Alimentos naturais de origem animal são ótimas fontes proteicas, de vitaminas e de minerais (BRASIL, 2014). O consumo de frutas e de verduras in natura garante 0 aporte da maioria dos micronutrientes e da fibra dietética. Ademais, pode ajudar a reduzir o consumo de alimentos ricos em gorduras saturadas, açucares, sal e dessa maneira contribuir para o estado nutricional adequado (NÚÑNZ et al., 2019). Enquanto os cereais contribuem para o apropriado teor de calorias e carboidratos complexos na dieta (BRASIL, 2014).

Cientes da importância da alimentação adequada durante a infância, enfatiza-se a influência do ambiente escolar sobre as preferências e escolhas das crianças, que tendem a ser mantidos nos demais ciclos da vida. Destaca-se a relevância das instituições de atendimento em período integral, espaços educativos que ofertam três ou mais refeições diárias, como medida de promoção da alimentação saudável. Visto que, propiciam uma maior disponibilidade dos alimentos in natura e minimamente processados, fator de relevância para a saúde pública (BENTO et al., 2018). 
O nutricionista é fundamental na execução e na garantia do alcance dos objetivos do programa. Bem como, na condução de ações de Educação Alimentar e Nutricional (EAN) com crianças, pais e professores, para a promoção de hábitos alimentares saudáveis, conscientização e valorização do PNAE como um dos eixos fundamentais para a garantia da SAN das crianças (ROCHA et al., 2018).

\section{CONSIDERAÇÕES FINAIS}

Os resultados demostram o parcial atendimento às recomendações nutricionais do PNAE e apontam para a necessidade de ajustes aos cardápios, aumentando a oferta de frutas, verduras, legumes, grãos integrais e proteína animal. Especificamente para atender as metas estabelecidas pelo programa quanto ao consumo de fibra e ferro, considerando as especificidades do público-alvo, a fim de garantir o adequado aporte nutricional.

Dentre as principais barreiras para que não se atinja os requerimentos nutricionais no programa, destacam-se o número insuficiente de nutricionistas no quadro técnico, a infraestrutura da instituição educacional (espaço físico), equipamentos, utensílios e recursos humanos e financeiros.

Enfatiza-se o papel fundamental do profissional nutricionista, responsável por planejar e garantir que o cardápio ofertado, contemple todos os nutrientes necessários, buscando, assim, a adequação e evitando carências ou excessos nutricionais.

Reforça-se a importância da participação da comunidade no controle social e no acompanhamento das ações para garantir a oferta da alimentação adequada às prerrogativas do PNAE.

Ademais, são necessários investimentos governamentais para a formulação, a implementação e a avaliação de políticas públicas que incentivem a promoção e a manutenção da alimentação adequada e saudável no ambiente escolar.

Evidenciamos a necessidade de outros estudos que investiguem a adequação dos cardápios às recomendações do PNAE, haja vista a importância do programa no atendimento das necessidades nutricionais e na formação dos hábitos alimentares adequados da clientela atendida.

\section{REFERÊNCIAS BIBLIOGRÁFICAS}

ABRANCHES, M.V.; PAULA, H.A.; MATA, G.M.; SALVADOR, B.C.; MARINHO, M.S.; PRIORE, S.E. Avaliação da adequação alimentar de creches pública e privada no contexto do programa nacional de alimentação escolar. Nutrire, v.34, n.2, p.43-57, 2009.

ALMEIDA, E.L.S.T.; FONTES, M.F.S.; ANJOS, A.V.; SANTOS, L.C.; PEREIRA, S.C.L. Alimentação em unidades de educação infantil: planejamento, processo produtivo, distribuição e adequação da 
refeição principal. O Mundo da Saúde, v.39, n.3, p.333-344, 2015.

BENTO, B.M.A.; MOREIRA, A.C.; CARMO, A.S.; SANTOS, L.C.; HORTA, P.M. A higher number of school meals is associated with a less-processed diet. Jornal de Pediatria, v.94, n.4, p.404-9, 2018.

BERNAUD, F.S.R.; RODRIGUES, T.C. Fibra alimentar - ingestão adequada e efeitos sobre a saúde do metabolismo. Arquivos Brasileiros de Endocrinologia \& Metabologia, v.57, n.6, p.397-405,2013.

BERTIN, R.L.; KARKLE, E.N.L.; ULBRICH, A.Z.; NETO, A.S.; BOZZA, R.; ARAUJO, I.Q.; CAMPOS, W. Estado nutricional e consumo alimentar de adolescentes da rede pública de ensino da cidade de São Mateus do Sul, Paraná, Brasil. Revista Brasileira Saúde Materno Infantil, v.8, n.4, p.435-43, 2008.

BIANCHINI, V.U.; MARTINELLI, S.S.; SOARES, P.; FABRI, R.K.; CAVALLI, S. B. Criteria adopted for school menu planning within the framework of the Brazilian School Feeding Program. Revista de Nutrição, v.33, n.e190197, p.1-13, 2020.

BLACK, R.E.; VICTORA, C.G.; WALKER, S.P.; BHUTTA, Z.A.; CHRISTIAN. P.; ONIS, M.; EZZATI, M.; GRANTHAMMCGREGOR, S.; KATZ, J.; MARTORELL,
R.; UAUY, R. Maternal and child undernutrition and overweight in lowincome and middle-income countries. Lancet, v.382, n.9890, p.427-451, 2013.

BRASIL. Presidência da República. Lei $\mathbf{n}^{\circ}$ 11.947, de 16 de junho de 2009. Dispõe sobre 0 atendimento da alimentação escolar e do Programa Dinheiro Direto na Escola aos alunos da educação básica. Brasília, DF: Presidência da República, 2009. Disponível em:

http://www.planalto.gov.br/ccivil 03/ ato20 07-2010/2009/lei/111947.htm. Acesso em: 05 fev. 2020.

BRASIL. Instituto Brasileiro de Geografia e Estatística (IBGE). Pesquisa de orçamentos familiares 2008-2009: tabela de composição nutricional dos alimentos consumidos no Brasil. Rio de Janeiro: IBGE, 2011.150p.

BRASIL. Ministério da Educação. Fundo Nacional de Desenvolvimento da Educação. Resolução/CD/FNDE n 26, de 17 de junho de 2013. Dispõe sobre o atendimento da alimentação escolar aos alunos da educação básica no âmbito do Programa Nacional de Alimentação Escolar-PNAE. Brasília, 2013. Disponível em: http://www.fnde.gov.br. Acesso em: 05 fev. 2020.

BRASIL. Lei no 12.982, de 28 de maio de 2014. Altera a Lei $n^{0}$ 11.947, de 16 de junho 
de 2009, para determinar o provimento de alimentação escolar adequada aos alunos portadores de estado ou de condição de saúde específica. Diário Oficial da União, Brasília, 28 de maio de 2014.

BRASIL. Ministério da educação. Fundo Nacional de Desenvolvimento da Educação. Conselho Nacional dos Procuradores Gerais do Ministério Público dos Estados, do Distrito Federal e da União. Cartilha Nacional da Alimentação Escolar. 2. ed. Brasília: DF, 2015. 88p.

BRASIL. Ministério da Educação. Fundo Nacional de Desenvolvimento da Educação. Resolução/CD/FNDE n ${ }^{\circ}$, de 08 de maio de 2020. Dispõe sobre o atendimento da alimentação escolar aos alunos da educação básica no âmbito do Programa Nacional de Alimentação Escolar - PNAE. Brasília, 2020a. Disponível em: https://www.fnde.gov.br. Acesso em: 05 jun. 2020.

BRASIL. Fundo Nacional de Desenvolvimento da Educação (FNDE). Ministério da Educação, 2020b. Disponível em: http://www.fnde.gov.br/. Acesso em: 05 mar. 2020.

CONCEIÇÃO, S.I.O.; SANTOS, C.J.N.; SILVA, A.A.M.; SILVA, J.S.; OLIVEIRA, TC. Consumo alimentar de escolares das redes pública e privada de ensino em São
Luís, Maranhão. Revista de Nutrição, v.23, n.6, p.993-1004,2010.

FERREIRA, H.S.; MOURA, R.M.M.; ASSUNÇÃO, M.L.; HORTA, B.L. Fatores associados à hipovitaminose $A$ em crianças menores de cinco anos. Revista Brasileira de Saúde Materno Infantil, v. 13, n.3, p.223-235, 2013.

FRAMPTON, A.M.; SISSON, S.B.; HORM, D.; CAMPBELL, J.E.; LORA, K.; LADNER, J.L. What's for Lunch? An Analysis of Lunch Menus in 83 Urban and Rural Oklahoma Child-care Centers providing allday care to preschool children. Journal of Academy of Nutrition Dietetics, v.114, n.9, p.1367-1374, 2014.

INOUE, D.Y.; OSÓRIO, M.M.; TACONELI, C.A.; SCHMIDT, S.T.; ALMEIDA, C.C.B. Consumo alimentar de crianças de 12 a 30 meses que frequentam Centros Municipais de Educação Infantil no município de Colombo, Sul do Brasil Revista de Nutrição, v.28, n.5, p.523-532, 2015.

ISSA, R.C.; MORAES, L.F.; FRANCISCO, R.R.J.; SANTOS, L.C.; ANJOS, A.F.V.; PEREIRA, S.C.L. Alimentação escolar: planejamento, produção, distribuição e adequação. Revista Panamericana de Salud Publica, v.35, n.2, p.96-103, 2014. 
LEAL, K.K.; SCHNEIDER, B.C.; FRANÇA, G.V.A.; GIGANTE, D.P.; SANTOS, I.; ASSUNÇÃO, M.C.F. Qualidade da dieta de pré-escolares de 2 a 5 anos residentes na área urbana da cidade de Pelotas, RS. Revista Paulista de Pediatria, v.33, n.3, p.311-318, 2015.

LEÃO, P.V.; DIAS, R.M.; FRAZÃO, A.G.F.; DIAS, I.A.; SILVA, I.R.P.; CORRÊA, N.A.F.; CAVALCANTI, C.D.T.D. Nutritional analysis of the School Feeding Program menus offered in a municipality of Pará. $\mathbf{0}$ Mundo da Saúde, v.42, n.1, p.181-198, 2018.

LOPES, J.M.L.; ZANELATO, E.F.N.; ROCHA, S.A.M.T.; FONSECA, C.S.; ALVES, R.D;M. Avaliação da Adequação de ferro, vitamina A e vitamina C da merenda ofertada a pré-escolares em um centro educacional público de Viçosa, MG. In: $7^{\circ}$ SIMPÓSIO DE PRODUÇÃO ACADÊMICA DA FACULDADE DE CIÊNCIAS BIOLÓGICAS E DA SAÚDE, 2015, Viçosa. Anais [...].Viçosa, 2015. p.100-5.

MOREIRA, A.; BENEDETTI, N.C.M.; SARON, M.L.G.; NEVES, A.S.; SOUZA, E.B. A influência do estado nutricional e da ingestão alimentar na aprendizagem escolar. Cadernos UniFOA, v.10, n.29, p.105-13, 2015.

NÚCLEO DE ESTUDOS E PESQUISAS EM ALIMENTAÇÃO. Universidade
Estadual de Campinas. Tabela brasileira de composição de alimentos - TACO. 4. ed. rev. e ampl. Campinas: Book Editora, 2011.

NÚÑEZ, A.; COLLANTE, C.; LÓPEZ, M. I.; GALEANO, C. Impacto de la estrategia de entrega de frutas com educación nutricional sobre el estado nutricional y consumo frutas en escolares de la escuela pública Héroes Luqueños de la comunidad de Jukyry Luque, Paraguay. Memorias del Instituto de Investigaciones en Ciencias de la Salud, v.17, n.3, p.55-62, 2019.

PACHECO; N.M.; CHAVES, A.C.L. Meu Gui@Alimentar Virtu@I. PUC Minas, MG., 2013. 86p. Disponível em: http://www1.pucminas.br/imagedb/docume nto/DOC DSC NOME ARQUI201509151 51119.pdf . Acesso em: 20 fev. 2020.

PAIVA, J.B.; FREITAS, M.C.S.; SANTOS, L.A.S. Significados da alimentação escolar segundo alunos atendidos pelo Programa Nacional de Alimentação Escolar. Ciência \& Saúde Coletiva, v.21, n.8, p. 2507-16, 2016.

PROENÇA, R.P.C.; SOUSA, A.A.; VEIROS, M.B.; HERING, B. Qualidade nutricional e sensorial na produção de refeições. Florianópolis: Editora da UFSC; 2008. 
RABELO, A.L.D.; CORREIA, R.P.; MOURA, C.S.S.; LIMA. R.T.S.; BICALHO, N.P.D.; SCHEREINER, I.M.O.; MARTINS, R.G. A merenda Chegou! Uma estratégia inovadora na produção e distribuição da merenda escolar em uma realidade ribeirinha amazônica. Saúde \&

Transformação Social, v.3, n.3, p.52-6, 2012.

ROCHA, N.P.; FILGUEIRAS, M.S.; ALBUQUERQUE, F.M.; MILAGRES, L.C.; CASTRO, A.P.P.; SILVA, M. A.; COSTA, G. D.; PRIORE, S.E.; NOVAES, J. F. Análise do programa nacional de alimentação escolar no município de Viçosa, MG, Brasil. Revista de Saúde Pública, v. 52, n.16, p.1-10, 2018.

SOCIEDADE BRASILEIRA DE PEDIATRIA. Departamento de Nutrologia. Obesidade na infância e adolescência Manual de Orientação. São Paulo: Sociedade Brasileira de Pediatria. Departamento de Nutrologia, 2008.116p.

SOUZA， C.A.N.; LONGO-SILVA， G.; MENEZES, R.C.E.; ARAUJO, A.C.; TOLONI, M.H.A.; OLIVEIRA, A. A. Adequação nutricional e desperdício de alimentos em Centros de Educação Infantil. Ciência \& Saúde Coletiva, v.23, n.12, p.4177-4188, 2018. 